Portland State University

PDXScholar

4-1-2009

\title{
Large-scale Assembly of Periodic Nanostructures with Metastable Square Lattices
}

\author{
Chih-Hung Sun \\ University of Florida \\ Wei-Lun Min \\ University of Florida \\ Nicholas C. Linn \\ University of Florida \\ Peng Jiang \\ University of Florida \\ Bin Jiang \\ Portland State University
}

Follow this and additional works at: https://pdxscholar.library.pdx.edu/mth_fac

Part of the Mathematics Commons

Let us know how access to this document benefits you.

\section{Citation Details}

Sun, Chih-Hung, Min, Wei-Lun, Linn, Nicholas, C., Jiang, Peng, and Jiang, Bin (2009) Large-scale Assembly of Periodic Nanostructures with Metastable Square Lattices. Journal of Vacuum Science \& Technology B, $27,1043-1057$

This Article is brought to you for free and open access. It has been accepted for inclusion in Mathematics and Statistics Faculty Publications and Presentations by an authorized administrator of PDXScholar. Please contact us if we can make this document more accessible: pdxscholar@pdx.edu. 


\title{
Large-scale assembly of periodic nanostructures with metastable square lattices
}

\author{
Chih-Hung Sun, Wei-Lun Min, Nicholas C. Linn, and Peng Jiang ${ }^{\text {a) }}$ \\ Department of Chemical Engineering, University of Florida, Gainesville, Florida 32611 \\ Bin Jiang \\ Department of Mathematics and Statistics, Portland State University, Portland, Oregon 97201
}

(Received 10 November 2008; accepted 16 March 2009; published 17 April 2009)

\begin{abstract}
This article reports a simple and scalable spin-coating technique for assembling non-close-packed colloidal crystals with metastable square lattices over wafer-scale areas. The authors observe the alternate formation of hexagonal and square diffraction patterns when the thickness of the colloidal crystals is gradually reduced during spin coating. No prepatterned templates are needed to induce the formation of the resulting metastable crystals with square arrangement. This bottom-up technology also enables the large-scale production of a variety of squarely ordered nanostructures that are consistent with the industry-standard rectilinear coordinate system for simplified addressing and circuit interconnection. Broadband moth-eye antireflection gratings with square lattices have been fabricated by using the shear-aligned colloidal monolayers as structural templates. Optical reflection measurement shows the squarely ordered arrays exhibit lower reflection than the nature-inspired hexagonal arrays with the similar structural parameters. (0) 2009 American Vacuum Society. [DOI: 10.1116/1.3117347]
\end{abstract}

\section{INTRODUCTION}

Large-scale fabrication of subwavelength periodic nanostructures is of great technological importance in developing new materials for photonic, electronic, magnetic, and biological applications. ${ }^{1-4}$ Various lithographic technologies (e.g., photolithography, electron-beam lithography, and interference lithography) have been extensively utilized in generating arbitrary nanostructures. However, these top-down techniques require sophisticated equipment and are expensive to implement. Bottom-up colloidal self-assembly and subsequent templating fabrication provide a much simpler and inexpensive alternative to nanolithography in creating a large variety of periodic nanostructures. ${ }^{5-7}$ Prominent applications of the templated materials include photonic crystals, ${ }^{6,8}$ surface-enhanced Raman scattering-enabled biosensors, ${ }^{5}$ and macroporous separation media and catalyst support, ${ }^{9}$ just to name a few. Unfortunately, current colloidal templating technologies suffer from low throughput and incompatibility with mature microfabrication, limiting the mass production and on-chip integration of practical devices. ${ }^{6}$ Furthermore, only hexagonally ordered colloidal crystals are available through traditional self-assembly, whereas less energetically favorable square arrays are compatible with semiconductor integrated circuit design standards. ${ }^{10}$ To fabricate colloidal arrays with metastable square lattices, template-assisted self-assembly has been developed. ${ }^{11,12}$ Nevertheless, commensurate trenches with well-controlled dimensions need to be created by lithographic techniques to confine colloidal particles within them.

We have recently developed a simple and scalable spincoating technology for assembling submicrometer-scale

$\overline{\text { a) Electronic mail: pjiang@che.ufl.edu }}$ silica particles into wafer-scale colloidal crystals with unusual non-close-packed structures. ${ }^{13}$ Both monolayer and multilayer crystals with large domain sizes can be fabricated by simply adjusting the spin-coating conditions. However, only easily obtained hexagonal arrays have been created by the spin-coating technology. We further demonstrate that the shear-aligned monolayer colloidal arrays can be utilized as etching masks during conventional reactive ion etching (RIE) processes to generate broadband moth-eye antireflection coatings (ARCs) on a number of substrates. ${ }^{14-16}$ Antireflection coatings are widely utilized in eliminating the "ghost images" for flat-panel displays, increasing the transmittance for optical lenses, improving the out-coupling efficiency of semiconductor light emitting diodes, and enhancing the conversion efficiency of solar cells. ${ }^{17-24}$ The severe reflection from an optical surface arises from the abrupt change in the refractive index at the interface. ${ }^{25}$ Moth-eye ARCs, which are inspired by the grainy microstructures on the corneas of moths consisting of a non-close-packed hexagonal array of conical nipples, can suppress reflection over a broader range of wavelengths and wider angles of incidence than traditional multilayer dielectric ARCs. ${ }^{26-28}$ Periodic arrays of subwavelength nipples generate a graded transition of refractive index between air and cornea, thus can greatly reduce reflection from the interface.

To fabricate subwavelength moth-eye ARCs, complex top-down technologies, such as electron-beam lithography ${ }^{28}$ and interference lithography, ${ }^{27}$ have been extensively employed. It is more straightforward to create square instead of nature-inspired hexagonal nipple arrays using these topdown techniques. To help design better moth-eye ARCs, it is desirable to compare the antireflective performance between the natural hexagonal and the technologically compatible square-structured moth-eye ARCs with the similar geometri- 
cal parameters. Our colloidal templating approach enables the formation of hexagonally ordered nipple arrays. ${ }^{14-16}$ However, the effect of crystalline structure on the antireflective performance of the templated moth-eye ARCs has not been systematically investigated due to the restricted formation of hexagonal crystals during spin coating. Here we report on the large-scale fabrication of squarely arranged colloidal monolayers with non-close-packed structures by the spin-coating technique. No prepatterned templates are required to induce the formation of these metastable arrays. The antireflection properties of the templated silicon pillar arrays with square and hexagonal lattices are also compared to evaluate the effect of crystalline structure on the performance of moth-eye ARCs.

\section{EXPERIMENT}

The fabrication of wafer-scale, monolayer, non-closepacked colloidal crystals with square lattices was performed by following the similar spin-coating procedures as reported in Ref. 13. In short, monodispersed silica colloids synthesized by the Stöber method ${ }^{29}$ were dispersed in ethoxylated trimethylolpropane triacrylate (ETPTA) (Sartomer) monomers to make final particle volume fraction of $\sim 20 \%$. $2 \%$ (weight) Darocur 1173 (Ciba Specialty Chemicals) was added as the photoinitiator. The silica-ETPTA suspension was dispensed on a 3-acryloxypropyl trichlorosilane (Gelest) primed (100) silicon wafer (Wafernet) and then spin coated on a standard spin coater (WS-400B-6NPP-Lite Spin Processor, Laurell). The typical recipe for spin coating $380 \mathrm{~nm}$ silica particles to obtain square-lattice crystals was $200 \mathrm{rpm}$ for $60 \mathrm{~s}, 300 \mathrm{rpm}$ for $60 \mathrm{~s}, 1000 \mathrm{rpm}$ for $30 \mathrm{~s}, 3000 \mathrm{rpm}$ for $10 \mathrm{~s}, 6000 \mathrm{rpm}$ for $10 \mathrm{~s}$, and $8000 \mathrm{rpm}$ for $150 \mathrm{~s}$. The spincoating process was stopped once a strong four-arm diffraction pattern was observed on the wafer surface and the monomer was then photopolymerized for $4 \mathrm{~s}$ using a pulsed UV curing system (Xenon). The polymer matrix was finally removed using a Unaxis Shuttlelock RIE/ICP reactive ion etcher operating at $40 \mathrm{mTorr}$ oxygen pressure, $40 \mathrm{SCCM}$ (SCCM denote cubic centimeters per minute at STP) flow rate, and $100 \mathrm{~W}$ for $120 \mathrm{~s}$ to release the embedded colloidal monolayers with square lattices.

The spin-coated colloidal monolayers were then used as etching masks during a chlorine RIE process $(5$ mTorr pressure, 20 SCCM chlorine flow rate, and $80 \mathrm{~W}$ ) to generate subwavelength silicon pillar arrays. The templating silica spheres were then removed by dissolving in a $2 \%(\mathrm{v} / \mathrm{v})$ hydrofluoric acid aqueous solution for $120 \mathrm{~s}$. An Ocean Optics HR4000 visible-near-IR spectrometer with a reflection probe was used for specular reflectance measurements. A calibrated halogen light source was used to illuminate the sample. Measurements were performed at normal incidence and the cone angle of collection was less than $5^{\circ}$. Final value of absolute reflectivity was the average of several measurements obtained from different spots on the sample surface. Scanning electron microscopy (SEM) was carried out on a JEOL 6335F FEG-SEM.

\section{RESULTS AND DISCUSSION}

The fabrication of wafer-scale, monolayer, non-closepacked colloidal crystals with square arrangement is similar to the spin-coating procedures, as reported in our previous publications. ${ }^{13}$ This methodology is based on shear-aligning concentrated colloidal suspensions with submicrometer-scale silica colloids dispersed in nonvolatile triacrylate monomers. Diffraction patterns from the shear-aligned colloidal crystals can be easily observed by illuminating white light on the samples during spin coating using a fiber-optic illuminator. When the spin-coating speed is low $(<6000 \mathrm{rpm})$, we only observe six-arm diffraction patterns with exact $60^{\circ}$ angles between neighboring arms, indicating the formation of hexagonally ordered colloidal crystals. ${ }^{13,30}$ Unexpected results occur when the spin speed is higher than $6000 \mathrm{rpm}$. We observe the alternate formation of hexagonal and square diffraction patterns when the thickness of the colloidal crystals is gradually reduced during spin coating. The spin-coating process is stopped once a strong four-arm diffraction pattern is formed on the wafer surface and the ETPTA monomers are then photopolymerized by exposure to UV radiation. The solidified ETPTA matrix can finally be removed by oxygen plasma etching to release the embedded colloidal arrays.

Figure 1(A) shows a photograph of a 4 in. diameter colloidal monolayer sample made from $380 \mathrm{~nm}$ silica spheres and spin coated at $8000 \mathrm{rpm}$ for $150 \mathrm{~s}$. The sample exhibits a distinctive four-arm diffraction pattern under white light illumination, and the angles between the neighboring diffraction arms are $90^{\circ}$. This pattern is a characteristic of longrange square ordering. This is confirmed by the SEM image in Fig. 1(B) and is further evidenced by the squarely arranged peaks in the Fourier transform of a low-magnification SEM image, as shown in the inset of Fig. 1(B). Further SEM characterization shows that the squarely arranged arrays cover the whole wafer surface, and the crystals are polycrystalline with typical domain size of several tens of micrometers. To obtain colloidal monolayers with larger singlecrystalline domains, the layer-by-layer thinning technology ${ }^{16}$ can be employed to gradually reduce the thickness of the spin-coated multilayer crystals, which usually exhibit better ordering than shear-aligned monolayer arrays.

The spin-coating technique enables the straightforward production of colloidal crystals with unusual non-closepacked structure, which is desirable for a number of nanomanufacturing applications (e.g., in creating non-closepacked moth-eye nipple arrays). ${ }^{26}$ The non-close-packed structure of the spin-coated square crystal is clearly evident from the SEM image in Fig. 1(B). The interparticle distance of the square crystal is determined to be $\sim 1.46 D$, where $D$ is the diameter of silica spheres, by the first peak of the pair correlation function (PCF) [Fig. 1(C)], $g(r)$, which is calculated from a low-magnification SEM image as

$$
g(r)=\frac{1}{\langle\rho\rangle} \frac{d n(r, r+d r)}{d a(r, r+d r)},
$$

where $\langle\rho\rangle$ is the average particle number density and $r$ is the particle radius. This interparticle distance is almost identical 


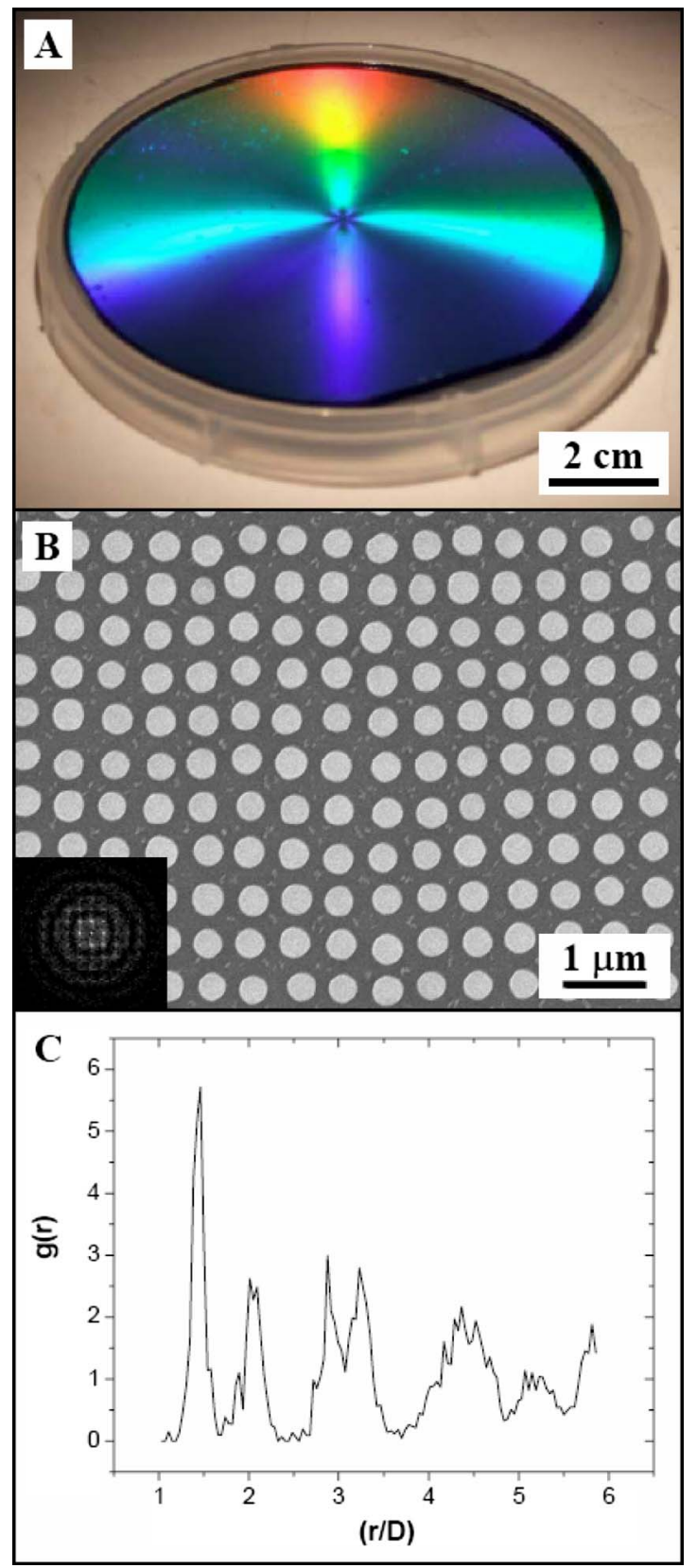

FIG. 1. (Color online) Spin-coated monolayer, non-close-packed colloidal crystal with metastable square lattice. (A) Photograph of a 4 in. sample illuminated with white light. (B) SEM image of the sample in (A). The inset showing the Fourier transform of a low-magnification image. (C) Pair correlation function calculated from a low-magnification SEM image. $380 \mathrm{~nm}$ silica spheres are used for making the sample.

to that of the shear-aligned hexagonal crystals $(\sim 1.41 D) .{ }^{13}$ The electrostatic repulsion between silica colloids could contribute to the observed non-close-packed structures. The zeta potential measurement shows that the silica particles are negatively charged $(\zeta \sim-40 \mathrm{mV})$ in ETPTA monomers.

Extensive spin-coating experiments show that the squarely arranged colloidal crystals are metastable structures between the more stable hexagonal crystals during the spin thinning process. Similar squarely arranged transitional structures have also been observed during colloidal convec-

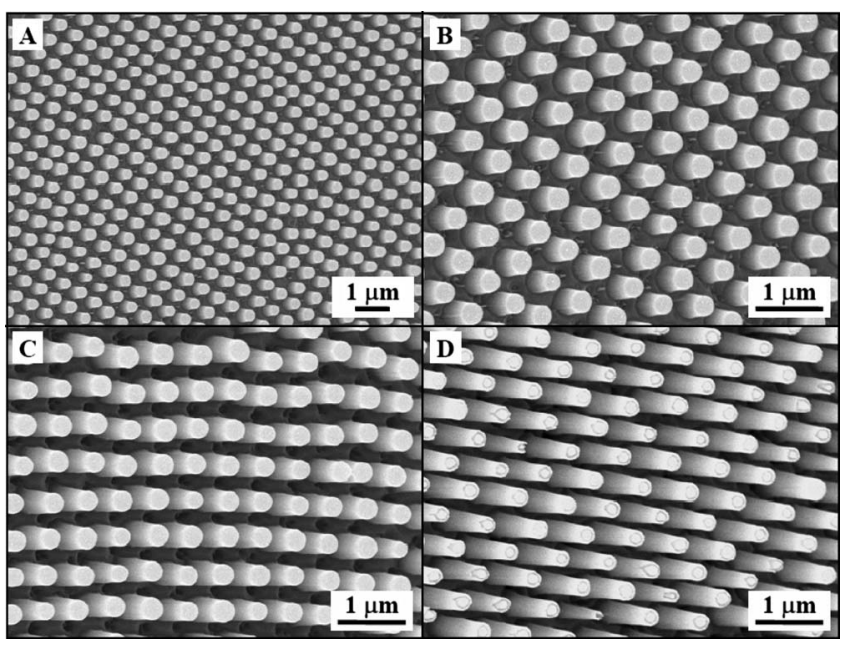

FIG. 2. Side-view SEM images of square-lattice silicon pillar arrays templated from spin-coated colloidal crystals at different RIE durations. (A) 15 min RIE. (B) Same sample as (A) but with higher magnification. (C) $30 \mathrm{~min}$ RIE. (D) $45 \mathrm{~min}$ RIE. $380 \mathrm{~nm}$ silica spheres are used for making these samples.

tive self-assembly ${ }^{31}$ and spin coating of colloids in volatile solvents, ${ }^{32}$ although these processes generate close-packed crystals. The detailed formation mechanism of the squarely arranged colloidal crystals during spin coating is still under investigation. We speculate that the high shear stress at high spin speed $(>6000 \mathrm{rpm})$ leads to the partial melting of the shear-aligned hexagonal crystals. ${ }^{33,34}$ It is well known that the microstructure for suspensions subjected to shear flow depends on the shear stress. ${ }^{35}$ The interactions of the applied hydrodynamic, Brownian, and the colloidal forces could induce the formation of less stable square crystals. ${ }^{36}$

Besides providing a simple and scalable bottom-up methodology for generating non-close-packed colloidal crystals with square ordering, the current spin-coating technology also enables the wafer-scale fabrication of a variety of squarely ordered nanostructures that are consistent with the industry-standard rectilinear coordinate system. ${ }^{10}$ Here we only demonstrate the fabrication of subwavelength moth-eye antireflection coatings on crystalline silicon substrates by using the shear-aligned square colloidal monolayers as templates. Other templating techniques developed for the hexagonal arrays in fabricating periodic nanostructures [e.g., nanohole arrays, ${ }^{37}$ attoliter microvials, ${ }^{38}$ and twodimensional (2D) magnetic nanodots] $]^{39}$ can also be easily extended to the spin-coated square arrays.

To create non-close-packed moth-eye pillar arrays, the squarely ordered colloidal monolayers [Fig. 1(B)] are utilized as etching masks during a chlorine RIE process. As the etching rate of silica is lower than that of silicon under the chlorine RIE conditions, silica particles protect the crystalline silicon substrate immediately underneath them from being etched, resulting in the formation of pillar arrays directly on the silicon surface. Figure 2 shows the tilted SEM images of square-lattice pillar arrays templated from $380 \mathrm{~nm}$ silica spheres and RIE etched for 15, 30, and 45 min, respectively. The preservation of the square ordering and the interparticle 


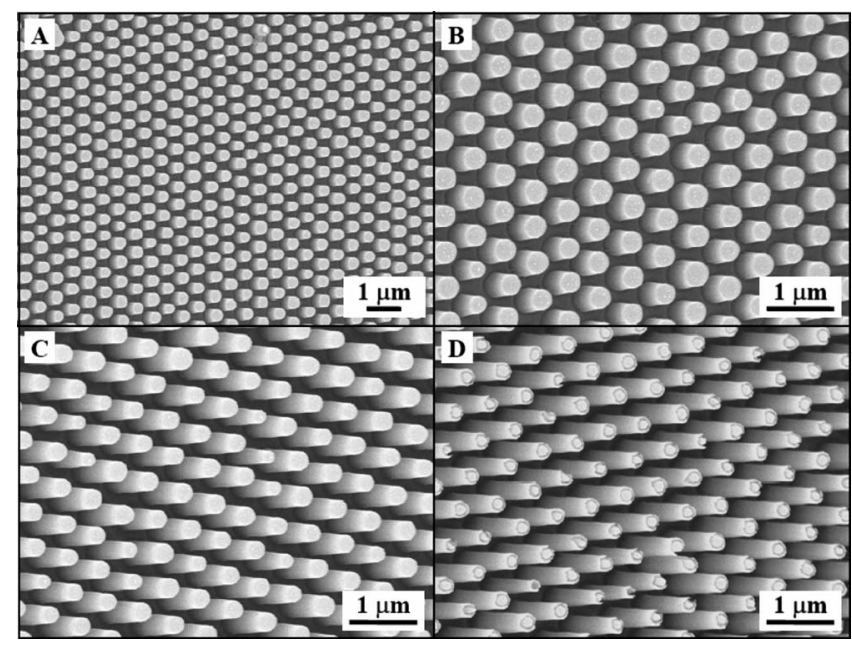

FIG. 3. Side-view SEM images of hexagonal-lattice silicon pillar arrays templated from spin-coated colloidal crystals at different RIE durations. (A) 15 min RIE. (B) Same sample as (A) but with higher magnification. (C) 30 min RIE. (D) 45 min RIE. $380 \mathrm{~nm}$ silica spheres are used for making these samples.

distance of the templating silica monolayers are obvious for the templated pillar arrays. It is also apparent that the depth of the templated pillars is controlled by the RIE duration, while the diameter of the silicon pillars is reduced for longer etching duration due to the isotropic characteristics of the RIE process. The shallow dimples formed on the top surfaces of the 45-min-etched pillars [Fig. 2(D)] are due to the shrinkage of the templating silica particles during the prolonged RIE etching that makes the particles too small to protect the underneath silicon from being partially etched.

The current spin-coating approach also enables us to compare the antireflective performance between the natureinspired hexagonal moth-eye arrays and the industrycompatible square arrays. For a fair comparison, the structural parameters of the hexagonal and square arrays of pillars (e.g., size, depth, shape, and interpillar distance) need to be identical. Figure 3 shows SEM images of hexagonal pillar arrays templated from hexagonally ordered colloidal monolayers consisting of $380 \mathrm{~nm}$ silica spheres. These samples are etched with their square counterparts (see Fig. 2) in the same RIE batches for 15, 30, and $45 \mathrm{~min}$, respectively. Except for the hexagonal ordering, the size, shape, and depth of the pillars are similar to those of the square-lattice pillars, as shown in Fig. 2. The PCF calculation shows the interpillar distance of the hexagonal array is $\sim 1.41 D$, close to that of the square array $(\sim 1.46 D)$.

The specular optical reflection of the templated silicon pillar arrays are evaluated using visible-near-IR reflectivity measurement at normal incidence. Figure 4 compares the optical reflection from the hexagonal- and square-lattice pillar arrays prepared by 9,15 , and 45 min RIE. All samples exhibit lower reflection than a bare silicon wafer, which shows $>35 \%$ reflectivity for most of the visible wavelengths. ${ }^{14}$ From Fig. 4, it is clearly seen that the reflectance spectra from the hexagonal and the square arrays are similar. The undulation of the reflection spectra are caused
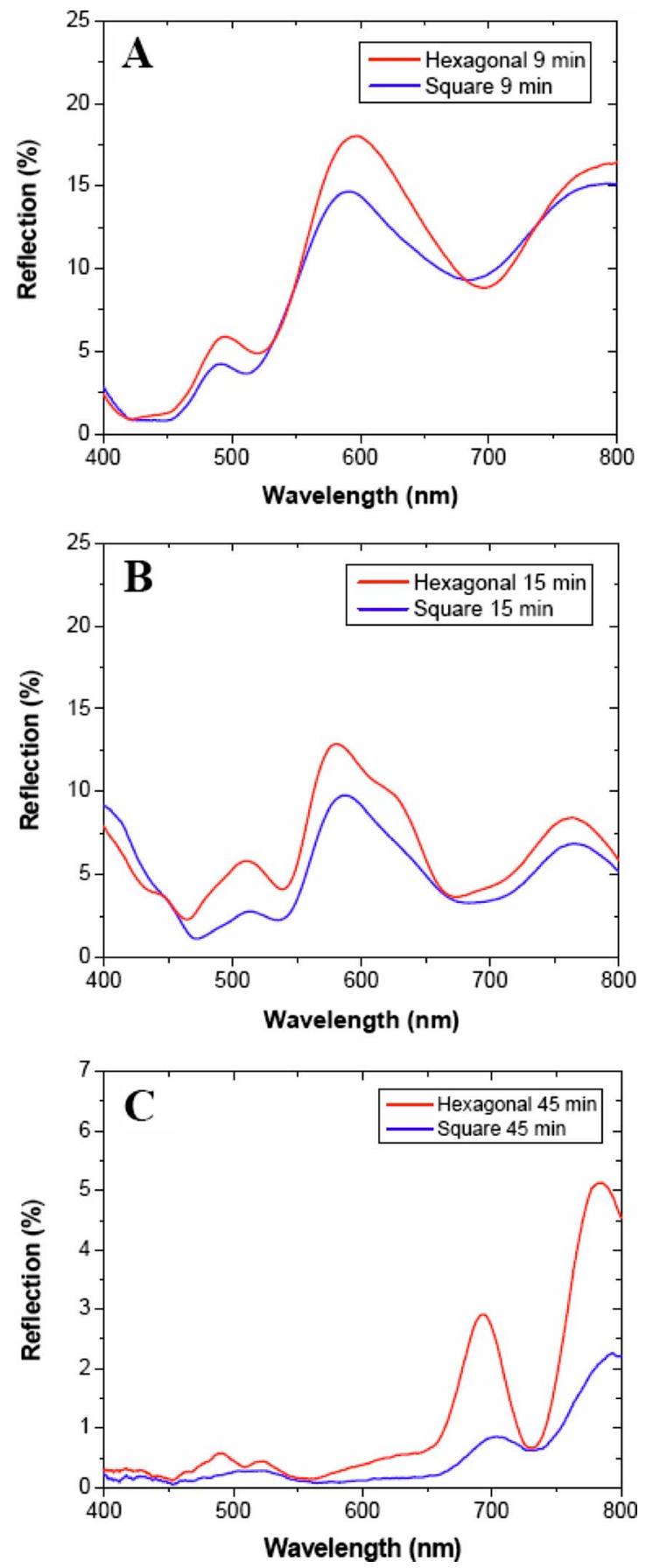

FIG. 4. (Color online) Comparison of the specular optical reflection at normal-incidence between the hexagonal- and square-lattice silicon pillar arrays templated from spin-coated colloidal crystals at different RIE durations: (A) 9 min RIE, (B) 15 min RIE, and (C) 45 min RIE. $380 \mathrm{~nm}$ silica spheres are used for making these samples.

by the interference of the incident and the reflected waves. ${ }^{25}$ In addition, the square arrays exhibit lower reflection than the hexagonal ones for all samples, although the difference is more significant for the taller pillars [Fig. 4(C)]. For the 45min-etched square array, the reflection is below $1 \%$ for all wavelengths below $750 \mathrm{~nm}$. This is the lowest reflection achieved so far for all samples prepared by our colloidal templating technique. ${ }^{14-16}$ We have also simulated the specu- 
lar reflection from the subwavelength moth-eye pillar arrays with both hexagonal and square structures using the rigorous coupled-wave analysis (RCWA) model. ${ }^{14,40}$ However, due to the limitation of our RCWA model, which assumes uniform refractive index for each sliced grating layer of the nipple arrays, ${ }^{14}$ the different crystalline structures have not been factored in the simulation. A more accurate RCWA model, where the refractive index of each sliced layer is going to be expanded as a 2D Fourier series, is under development to provide better understanding of the effect of the crystalline structure on the performance of moth-eye antireflection coatings.

\section{CONCLUSIONS}

We have developed a simple spin-coating technique for fabricating non-close-packed colloidal monolayers with metastable square lattices. Wafer-scale crystals can be rapidly assembled without the need for the prepatterned templates to induce the formation of the metastable arrays. We have also demonstrated that the shear-aligned square colloidal arrays can be used as structural templates to create subwavelength moth-eye antireflection gratings. The antireflection properties of the templated square-lattice subwavelength ARCs are compared to that of nature-inspired hexagonal arrays by specular reflection measurements. The squarely ordered ARCs exhibit lower reflection than the hexagonal arrays with similar structural parameters. This study could provide new insights into the optimal design and fabrication of efficient broadband ARCs for crystalline silicon solar cells.

\section{ACKNOWLEDGMENTS}

This work was supported in part by the National Science Foundation under Grant Nos. CBET-0651780 and CBET0744879, ACS Petroleum Research Fund, and the UF Research Opportunity Incentive Seed Fund.

${ }^{1}$ C. Genet and T. W. Ebbesen, Nature (London) 445, 39 (2007).

${ }^{2}$ J. G. C. Veinot, H. Yan, S. M. Smith, J. Cui, Q. L. Huang, and T. J. Marks, Nano Lett. 2, 333 (2002)

${ }^{3}$ S. H. Sun, C. B. Murray, D. Weller, L. Folks, and A. Moser, Science 287, 1989 (2000).

${ }^{4}$ M. J. Levene, J. Korlach, S. W. Turner, M. Foquet, H. G. Craighead, and
W. W. Webb, Science 299, 682 (2003).

${ }^{5}$ C. L. Haynes and R. P. Van Duyne, J. Phys. Chem. B 105, 5599 (2001).

${ }^{6}$ Y. A. Vlasov, X. Z. Bo, J. C. Sturm, and D. J. Norris, Nature (London) 414, 289 (2001).

${ }^{7}$ Y. N. Xia, B. Gates, Y. D. Yin, and Y. Lu, Adv. Mater. (Weinheim, Ger.) 12, 693 (2000).

${ }^{8}$ A. Blanco et al., Nature (London) 405, 437 (2000).

${ }^{9}$ B. T. Holland, C. F. Blanford, and A. Stein, Science 281, 538 (1998).

${ }^{10}$ C. B. Tang, E. M. Lennon, G. H. Fredrickson, E. J. Kramer, and C. J. Hawker, Science 322, 429 (2008).

${ }^{11}$ Y. D. Yin and Y. N. Xia, Adv. Mater. (Weinheim, Ger.) 14, 605 (2002).

${ }^{12}$ G. A. Ozin and S. M. Yang, Adv. Funct. Mater. 11, 95 (2001).

${ }^{13}$ P. Jiang and M. J. McFarland, J. Am. Chem. Soc. 126, 13778 (2004).

${ }^{14}$ C. H. Sun, P. Jiang, and B. Jiang, Appl. Phys. Lett. 92, 061112 (2008).

${ }^{15}$ W. L. Min, A. P. Betancourt, P. Jiang, and B. Jiang, Appl. Phys. Lett. 92, 141109 (2008).

${ }^{16}$ C. H. Sun, B. J. Ho, B. Jiang, and P. Jiang, Opt. Lett. 33, 2224 (2008).

${ }^{17}$ P. Lalanne and G. M. Morris, Nanotechnology 8, 53 (1997).

${ }^{18}$ S. Chattopadhyay, L. C. Chen, and K. H. Chen, Crit. Rev. Solid State Mater. Sci. 31, 15 (2006).

${ }^{19}$ S. Walheim, E. Schaffer, J. Mlynek, and U. Steiner, Science 283, 520 (1999).

${ }^{20}$ B. G. Prevo, E. W. Hon, and O. D. Velev, J. Mater. Chem. 17, 791 (2007).

${ }^{21}$ Y. F. Huang et al., Nat. Nanotechnol. 2, 770 (2007).

${ }^{22}$ U. Schulz, Appl. Opt. 45, 1608 (2006).

${ }^{23}$ J. Q. Xi, M. F. Schubert, J. K. Kim, E. F. Schubert, M. F. Chen, S. Y. Lin, W. Liu, and J. A. Smart, Nat. Photonics 1, 176 (2007).

${ }^{24}$ M. Ibn-Elhaj and M. Schadt, Nature (London) 410, 796 (2001).

${ }^{25}$ H. A. Macleod, Thin-Film Optical Filters, 3rd ed. (Institute of Physics Publishing, Bristol, 2001).

${ }^{26}$ D. G. Stavenga, S. Foletti, G. Palasantzas, and K. Arikawa, Proc. R. Soc. London, Ser. B 273, 661 (2006).

${ }^{27}$ A. Gombert, W. Glaubitt, K. Rose, J. Dreibholz, B. Blasi, A. Heinzel, D. Sporn, W. Doll, and V. Wittwer, Thin Solid Films 351, 73 (1999).

${ }^{28}$ Y. Kanamori, K. Kobayashi, H. Yugami, and K. Hane, Jpn. J. Appl. Phys., Part 1 42, 4020 (2003)

${ }^{29}$ W. Stober, A. Fink, and E. Bohn, J. Colloid Interface Sci. 26, 62 (1968).

${ }^{30}$ P. Pieranski, Contemp. Phys. 24, 25 (1983).

${ }^{31}$ L. L. Meng, H. Wei, A. Nagel, B. J. Wiley, L. E. Scriven, and D. J. Norris, Nano Lett. 6, 2249 (2006).

${ }^{32}$ A. Mihi, M. Ocana, and H. Miguez, Adv. Mater. (Wienheim, Ger.) 18, 2244 (2006).

${ }^{33}$ B. J. Ackerson and N. A. Clark, Phys. Rev. A 30, 906 (1984).

${ }^{34}$ Y. D. Yan and J. K. G. Dhont, Physica A 198, 78 (1993).

${ }^{35}$ J. Vermant and M. J. Solomon, J. Phys.: Condens. Matter 17, R187 (2005).

${ }^{36}$ R. L. Hoffman, Trans. Soc. Rheol. 16, 155 (1972).

${ }^{37}$ P. Jiang and M. J. McFarland, J. Am. Chem. Soc. 127, 3710 (2005).

${ }^{38}$ P. Jiang, Chem. Commun. (Cambridge), 1699 (2005).

${ }^{39}$ P. Jiang, Langmuir 22, 3955 (2006).

${ }^{40}$ M. G. Moharam, D. A. Pommet, E. B. Grann, and T. K. Gaylord, J. Opt. Soc. Am. A 12, 1077 (1995). 